\title{
Migrated guidewire: an unusual cause for recurrent aural polyps
}

\begin{abstract}
Aural polyps are secondary to multiple ear pathologies, most commonly inflammatory or cholesteatoma related. Here, we present a rare case of recurrent aural polyps caused by guidewire migration into the middle ear with serious systemic complications and our attempts at removal.
\end{abstract}

Keyword: Aural polyp; Bacteremia; Computed tomography; Guidewire 